\title{
OPTIMAL CONTROL FOR DISTRIBUTED SYSTEMS SUBJECT TO NULL-CONTROLLABILITY. APPLICATION TO DISCRIMINATING SENTINELS
}

\author{
OUSSEYNOU NAKOULimA ${ }^{1}$
}

\begin{abstract}
We consider a distributed system in which the state $q$ is governed by a parabolic equation and a pair of controls $v=(h, k)$ where $h$ and $k$ play two different roles: the control $k$ is of controllability type while $h$ expresses that the state $q$ does not move too far from a given state.

Therefore, it is natural to introduce the control point of view. In fact, there are several ways to state and solve optimal control problems with a pair of controls $h$ and $k$, in particular the Least Squares method with only one criteria for the pair $(h, k)$ or the Pareto Optimal Control for multicriteria problems.

We propose here to use the notion of Hierarchic Control. This notion assumes that we have two controls $h, k$ where $h$ will be the leader while $k$ will be the follower. The main tool used to solve the null-controllability problem with constraints on the follower is an observability inequality of Carleman type which is "adapted" to the constraints.

The obtained results are applied to the sentinels theory of Lions [Masson (1992)].
\end{abstract}

Mathematics Subject Classification. 35K05, 35K15, 35K20, 49J20, 93B05.

Received March 23, 2004.

Published online September 5, 2007.

\section{INTRODUCTION}

For $d \in \mathbf{N}^{*}$, let $\Omega$ be a bounded open subset of $\mathbf{R}^{d}$ with boundary $\Gamma$ of class $C^{2}, T>0$, and let $\omega$ be an open non empty subset of $\Omega$. Set $Q=\Omega \times(0, T), \Sigma=\Gamma \times(0, T), U=\omega \times(0, T)$. We consider the parabolic evolution equation:

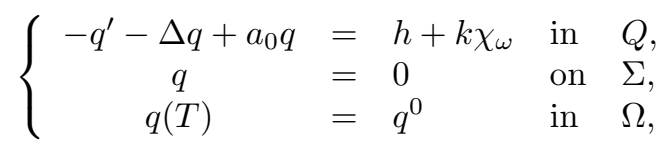

where (.)' is the partial derivative with respect to time $t, a_{0} \in L^{\infty}(Q), v=(h, k) \in L^{2}(Q) \times L^{2}(U), q^{0} \in L^{2}(\Omega)$ and $\chi_{\omega}$ denotes the characteristic function of $\omega$. It is well known that problem (1) admits a unique solution $q$ in the following Hilbert space (see for instance $[13,15]$ ):

$$
W(0, T)=\left\{\varphi \quad \mid \quad \varphi \in L^{2}\left(0, T ; H_{0}^{1}(\Omega)\right), \varphi^{\prime} \in L^{2}\left(0, T ; H^{-1}(\Omega)\right)\right\}
$$

Keywords and phrases. Heat equation, optimal control, controllability, Carleman inequalities, sentinels.

1 Université Antilles-Guyane, Département de Mathématiques et Informatique, 97159 Pointe-à-Pitre, Guadeloupe, France; onakouli@univ-ag.fr 
endowed with the natural norm

$$
\|\varphi\|_{W(0, T)}=\left\{\|\varphi\|_{L^{2}\left(0, T ; H_{0}^{1}(\Omega)\right)}^{2}+\left\|\varphi^{\prime}\right\|_{L^{2}\left(0, T ; H^{-1}(\Omega)\right)}^{2}\right\}^{\frac{1}{2}} .
$$

Remark 1. System (1) is a backward parabolic problem. It appears under this form in the sentinels theory of Lions as the associated adjoint state. (cf. [14], p. 22. See also below in Sect. 5).

We will use the notation

$$
q=q(x, t ; v)
$$

to mean that the solution $q$ of (1) depends of the control pair $v=(h, k)$ which plays a particular role. Roughly speaking, we would like to choose $v=(h, k)$ in order to achieve two objectives. The first one is formulated as follows: let $h$ be a given function in $L^{2}(Q)$ and

$$
\mathcal{K} \text { a real closed vector subspace of } L^{2}(U) .
$$

Denoting by $\mathcal{K}^{\perp}$ the orthogonal subspace of $\mathcal{K}$ in $L^{2}(U)$ we look for a control variable $k \in L^{2}(U)$ so that

$$
k \in \mathcal{K}^{\perp}
$$

and such that if $q=q(x, t ; v)$ is the unique solution of (1), then

$$
q(., 0 ; v)=0 \quad \text { in } \quad \Omega .
$$

The role of $k$ is to guarantee the null-controllability property (5) in the presence of the forcing term $h$ and under the restriction (4).

Remark 2. The null-controllability problem (1), (4) and (5) is by now well understood in the case $\mathcal{K}=\{0\}$. It has been studied by several authors using different methods. We refer to Russell [17], Lebeau and Robbiano [12], Fursikov and Imanuvilov [10]. We also refer to Barbu [1], Doubova et al. [5], Fabre et al. [6], Fernández-Cara [7], Fernández-Cara and Guerrero [8], Zuazua [18,20], and their bibliography for other related controllability problems.

The null-controllability problem (1), (4) and (5) in the case $\mathcal{K} \neq\{0\}$ has been studied by the author in [16] when $\mathcal{K}$ is of finite dimension. In this case, some compatibility conditions are requiered for controllability to hold. We shall return to this matter latter on.

In addition to the null-controllability property (5) subject to the constraint (4), the second goal is to choose the forcing term $h$ such that (4) and (5) are satisfied and that, also,

$$
q(., . ; v) \text { is not too far from } z_{d},
$$

where $z_{d}$ is given in $Q$.

In order to make these objectives more precise, we introduce a suitable non-negative weight function $\theta$ which will be defined below and consider the space

$$
L_{\theta}^{2}(Q)=\left\{h \mid h \in L^{2}(Q), \quad \theta h \in L^{2}(Q)\right\},
$$

a Hilbert space for the scalar product and norm

$$
(h, \ell)_{\theta}=\int_{Q} \theta^{2} h \ell \mathrm{d} x \mathrm{~d} t, \quad\|h\|_{\theta}=\|\theta h\|_{L^{2}(Q)} .
$$


For $h \in L_{\theta}^{2}(Q)$, we will see that there exist several controls $k$ such that (1), (4) and (5) are satisfied. Therefore, we need to add some criteria to select $k$. More precisely, for each $h$, we can consider $\hat{k}_{\theta}(h)$ as being the control $k$ of minimal norm. Thus, we can define a mapping

$$
\hat{k}_{\theta}: h \mapsto \hat{k}_{\theta}(h)
$$

from $L_{\theta}^{2}(Q)$ into $L^{2}(U)$ such that $\hat{k}_{\theta}(h)$ satisfies (1), (4) and (5). Then, we define

$$
\hat{q}_{\theta}(h)=q\left(., . ;\left(h, \hat{k}_{\theta}(h)\right)\right),
$$

and introduce the weighted cost function $J_{\theta}$ defined as follows: for any control $h \in L_{\theta}^{2}(Q)$,

$$
J_{\theta}(h)=\frac{\alpha}{2}\left\|\hat{\theta}_{\theta}(h)-z_{d}\right\|_{L^{2}(Q)}^{2}+\frac{\beta}{2}\left\|\hat{k}_{\theta}(h)-k_{d}\right\|_{L^{2}(U)}^{2}+\frac{N}{2}\|h\|_{\theta}^{2}
$$

where $z_{d} \in L^{2}(Q), k_{d} \in L^{2}(U), \alpha, \beta \geq 0$ and $N>0$ are given.

Let us now consider the set of admissible controls

$$
\mathcal{U}_{a d}=\text { non empty closed convex subset of } L_{\theta}^{2}(Q) .
$$

Then, the second objective, briefly presented above, is achieved and made precise through the minimization problem

$$
\min J_{\theta}(h), \quad h \in \mathcal{U}_{a d} .
$$

If the minimum exists, we say that $h$ is the optimal leader, $k$ is the optimal follower and $q$ is the optimal state of the system.

The optimal control problem (8) is not standard because of the restriction (4), but also and mainly because of (5). The problem (8) has been studied by Dorville [3], and by Dorville et al. in [4] in the case $\mathcal{K}=\{0\}$. This article seems to be the first one dealing with the case $\mathcal{K} \neq\{0\}$.

The paper is organized as follows: Section 2 is devoted to define the optimal follower. The main tool is a constraint-adapted observability inequality given by Lemma 1. In Section 3, we consider the optimal control problem defined by (8), and prove the existence of a unique optimal control (Th. 2) and we give the optimality system for the optimal control (Th. 3). In Section 5, we give an application of the above results to the sentinels theory of Lions as revisited in [16].

\section{Preliminaries}

\subsection{Classical Carleman's inequality}

It is now well known that the null controllability analysis of parabolic equations is equivalent to the observability inequality of the associated adjoint state which is obtained by appropriate Carleman estimates. The main contributions in this area are due to Imanuvilov, who developed the use of Carleman estimates in the context of null controllability [11].

In order to state Carleman's inequality, we introduce now some objects and notations. Choose first some auxiliary function $\psi \in C^{2}(\bar{\Omega})$ which satisfies the following conditions:

$$
\psi(x)>0 \quad \forall x \in \Omega, \quad \psi(x)=0 \quad \forall x \in \Gamma, \quad|\nabla \psi(x)| \neq 0 \quad \forall x \in \overline{\Omega-\omega},
$$

Such a function $\psi$ exists according to Fursikov and Imanuvilov [10]. 
For any positive parameter $\lambda$ define then the following weight functions

$$
\varphi(x, t)=\frac{\mathrm{e}^{\lambda \psi(x)}}{t(T-t)}, \quad \eta(x, t)=\frac{\mathrm{e}^{2 \lambda\|\psi\|_{\infty}}-\mathrm{e}^{\lambda \psi(x)}}{t(T-t)},
$$

and adopt the following notations

$$
\left\{\begin{array}{l}
L_{0}=\frac{\partial}{\partial t}-\Delta, \\
L=\frac{\partial}{\partial t}-\Delta+a_{0} I, \\
\mathcal{V}=\left\{\rho \in C^{\infty}(\bar{Q}), \rho=0 \text { on } \Sigma\right\}
\end{array}\right.
$$

where $a_{0} \in L^{\infty}(Q)$. Now the inequality can be formulated as follows. There exist three constants $\lambda_{0}=$ $\lambda_{0}(\Omega, \omega)>1, s_{0}=s_{0}(\Omega, \omega, T)>1$ and $C=C(\Omega, \omega)>0$ such that for any $\lambda \geq \lambda_{0}$, any $s \geq s_{0}$ and for any $\rho \in \mathcal{V}$ the following inequality holds:

$$
\begin{aligned}
\int_{Q} \frac{\mathrm{e}^{-2 s \eta}}{s \varphi}\left(\left|\rho^{\prime}\right|^{2}+|\Delta \rho|^{2}\right) \mathrm{d} x \mathrm{~d} t+\int_{Q} s \lambda^{2} \varphi \mathrm{e}^{-2 s \eta} & |\nabla \rho|^{2} \mathrm{~d} x \mathrm{~d} t+\int_{Q} s^{3} \lambda^{4} \varphi^{3} \mathrm{e}^{-2 s \eta}|\rho|^{2} \mathrm{~d} x \mathrm{~d} t \\
& \leq C\left(\int_{Q} \mathrm{e}^{-2 s \eta}\left|L_{0}\right|^{2} \mathrm{~d} x \mathrm{~d} t+\int_{U} s^{3} \lambda^{4} \varphi^{3} \mathrm{e}^{-2 s \eta}|\rho|^{2} \mathrm{~d} x \mathrm{~d} t\right)
\end{aligned}
$$

The above inequality is referred to as the global Carleman inequality (see [10] and [11]). As $L_{0}=L-a_{0} I$, then from the previous inequality (10) we deduce another inequality for the operator $L$ by direct substitution in (10). We conclude the existence of three constants $\lambda_{1}=\lambda_{1}\left(\Omega, \omega, a_{0}\right)>1, s_{1}=s_{1}\left(\Omega, \omega, T, a_{0}\right)>1$ and $C=C(\Omega, \omega)>0$ such that for any $\lambda \geq \lambda_{1}$, any $s \geq s_{1}$ and for any $\rho \in \mathcal{V}$ the next inequality holds:

$$
\begin{aligned}
\int_{Q} \frac{\mathrm{e}^{-2 s \eta}}{s \varphi}\left(\left|\rho^{\prime}\right|^{2}+|\Delta \rho|^{2}\right) \mathrm{d} x \mathrm{~d} t+\int_{Q} s \lambda^{2} \varphi \mathrm{e}^{-2 s \eta} & |\nabla \rho|^{2} \mathrm{~d} x \mathrm{~d} t+\int_{Q} s^{3} \lambda^{4} \varphi^{3} \mathrm{e}^{-2 s \eta}|\rho|^{2} \mathrm{~d} x \mathrm{~d} t \\
& \leq C\left(\int_{Q} \mathrm{e}^{-2 s \eta}|L \rho|^{2} \mathrm{~d} x \mathrm{~d} t+\int_{U} s^{3} \lambda^{4} \varphi^{3} \mathrm{e}^{-2 s \eta}|\rho|^{2} \mathrm{~d} x \mathrm{~d} t\right)
\end{aligned}
$$

Since $\varphi$ does not vanish, we may set

$$
\theta=\frac{\mathrm{e}^{s \eta}}{\varphi \sqrt{\varphi}} \quad \text { so } \quad \frac{1}{\theta}=\varphi \sqrt{\varphi} \mathrm{e}^{-s \eta}
$$

Then $\theta \in C^{2}(Q)$ and $1 / \theta$ is bounded. By substitution in (10) the following inequality holds

$$
\int_{Q} \frac{1}{\theta^{2}}|\rho|^{2} \mathrm{~d} x \mathrm{~d} t \leq C\left(\int_{Q} \frac{1}{\theta^{2} \varphi^{3} s^{3} \lambda^{4}}|L \rho|^{2} \mathrm{~d} x \mathrm{~d} t+\int_{U} \frac{1}{\theta^{2}}|\rho|^{2} \mathrm{~d} x \mathrm{~d} t\right) .
$$

As a consequence of the boundeness of $1 / \theta$ and $1 / \varphi^{3} s^{3} \lambda^{4}$, the following inequality holds too:

$$
\int_{Q} \frac{1}{\theta^{2}}|\rho|^{2} \mathrm{~d} x \mathrm{~d} t \leq C\left(\int_{Q}|L \rho|^{2} \mathrm{~d} x \mathrm{~d} t+\int_{U}|\rho|^{2} \mathrm{~d} x \mathrm{~d} t\right) .
$$

All these results are by now well understood. We refer, for instance, to Fernández-Cara and Zuazua [9]. 


\subsection{Carleman's inequality adapted to linear constraints}

We are now concerned with a new observability inequality needed to address the problem that motivates this article. Indeed, for the null controllability problem with constraints, we need another observability inequality with partial measurements. More precisely, in order to deal with the constraint (4) we have to derive a more precise observability inequality adapted to the subspace $\mathcal{K}$ in (3). Assume that

$\mathcal{K}$ is finite dimensional,

and

any function $k \in \mathcal{K}$ such that $L k=0$ in $U$ is identically zero in $U$.

Denote by

and for $\rho \in L^{2}(Q)$

$$
P=\text { the orthogonal projection operator from } L^{2}(U) \text { onto } \mathcal{K}
$$

$$
P \rho=\text { the orthogonal projection of } \rho \chi_{\omega} .
$$

The following lemma is the key ingredient for our results.

Lemma 1. Assume that (13) and (14) hold. Then there exists a positive constant $C=C(\Omega, \omega)$ such that for any $\rho \in \mathcal{V}$ :

$$
\int_{Q} \frac{1}{\theta^{2}}|\rho|^{2} \mathrm{~d} x \mathrm{~d} t \leq C\left(\int_{Q}|L \rho|^{2} \mathrm{~d} x \mathrm{~d} t+\int_{U}|\rho-P \rho|^{2} \mathrm{~d} x \mathrm{~d} t\right) .
$$

Remark 3. The assumption (14) has been already introduced by Lions in [14], p. 33. Here is some case where this assumption is satisfied. As an example, consider the case in which each $m_{i}$ has its support in domains such as $\omega_{i} \times(0, T)$ with $\omega_{i} \subset \omega$ and $\omega_{i} \cap \omega_{j}=\emptyset$ for $i \neq j$. Assuming that $L m_{i} \neq 0$, then if $k \in \mathcal{K}$ and $L k=0$ in $\omega \times(0, T)$ we have $k=0$ in $\omega \times(0, T)$, and the assumption (14) is satisfied.

Proof of Lemma 1. The proof uses a well known compactness-uniqueness argument. Indeed, suppose that (17) does not hold. Then

$$
\left\{\begin{array}{l}
\forall n \in N^{*}, \exists \rho_{n} \in \mathcal{V}, \int_{Q} \frac{1}{\theta^{2}}\left|\rho_{n}\right|^{2} \mathrm{~d} x \mathrm{~d} t=1, \\
\int_{Q}\left|L \rho_{n}\right|^{2} \mathrm{~d} x \mathrm{~d} t \leq \frac{1}{n} \text { and } \int_{U}\left|\rho_{n}-P \rho_{n}\right|^{2} \mathrm{~d} x \mathrm{~d} t \leq \frac{1}{n} .
\end{array}\right.
$$

The proof consists in showing that (18) yields a contradiction. We do it in four steps.

(1) We have

$$
\int_{U} \frac{1}{\theta^{2}}\left|P \rho_{n}\right|^{2} \mathrm{~d} x \mathrm{~d} t \leq \int_{U} \frac{1}{\theta^{2}}\left|\rho_{n}\right|^{2} \mathrm{~d} x \mathrm{~d} t+\int_{U} \frac{1}{\theta^{2}}\left|\rho_{n}-P \rho_{n}\right|^{2} \mathrm{~d} x \mathrm{~d} t .
$$

Since $1 / \theta^{2}$ is bounded, it follows from (18),

$$
\int_{U} \frac{1}{\theta^{2}}\left|P \rho_{n}\right|^{2} \mathrm{~d} x \mathrm{~d} t \leq C
$$

Since $P \rho_{n} \in \mathcal{K}$ and $\mathcal{K}$ is finite dimensional, $P \rho_{n}$ (and so $\rho_{n}$ ) is bounded in $L^{2}(U)$.

(2) We can extract a subsequence, still denoted $\left(\rho_{n}\right)_{n}$, such that on the one hand

$$
\rho_{n} \rightarrow g \text { weakly in } L^{2}(U),
$$

and on the other hand:

$$
\rho_{n}-P \rho_{n} \rightarrow 0 \text { strongly in } L^{2}(U)
$$


Next, we deduce from the compactness of $P$ (because $\mathcal{K}$ is of finite dimension) that there exists $\sigma \in \mathcal{K}$ such that

$$
P \rho_{n} \rightarrow \sigma \text { strongly in } L^{2}(U) .
$$

We deduce from (21) and (22) that $\rho_{n} \rightarrow g=\sigma$ strongly in $L^{2}(U)$. Thanks to the continuity of $P$, we have $P \rho_{n} \rightarrow P g$ strongly in $L^{2}(U)$. Therefore, $P g=g$ and $g \in \mathcal{K}$.

(3) In fact, we have $g=0$. Indeed, from (18), we also have $L \rho_{n} \rightarrow 0$ strongly in $L^{2}(Q)$. Thus $L \rho_{n} \rightarrow 0$ strongly in $L^{2}(U)$. We deduce that $L \rho_{n} \rightarrow 0$ weakly in $\mathcal{D}^{\prime}(U)$ and so $L g=0$. The assumption (14) implies $g=0$ on $U$. Finally, $\rho_{n} \rightarrow 0$ strongly in $L^{2}(U)$.

(4) Since $\rho_{n} \in \mathcal{V}$, it follows from the observability inequality (12) that

$$
\int_{Q} \frac{1}{\theta^{2}}\left|\rho_{n}\right|^{2} \mathrm{~d} x \mathrm{~d} t \leq C\left(\int_{Q}\left|L \rho_{n}\right|^{2} \mathrm{~d} x \mathrm{~d} t+\int_{U}\left|\rho_{n}\right|^{2} \mathrm{~d} x \mathrm{~d} t\right) .
$$

Then, from the conclusions in the third step, we deduce that $\int_{Q} \frac{1}{\theta^{2}}\left|\rho_{n}\right|^{2} \mathrm{~d} x \mathrm{~d} t \rightarrow 0$ when $n \rightarrow+\infty$. The contradiction occurs thanks to the first condition in (18), where $\int_{Q} \frac{1}{\theta^{2}}\left|\rho_{n}\right|^{2} \mathrm{~d} x \mathrm{~d} t=1$. The proof of (17) is complete.

Corollary 1. Assume that (13) and (14) hold. Then there exists a positive constant $C=C\left(\Omega, \omega, a_{0}, T\right)$ such that for any $\rho \in \mathcal{V}$ :

$$
\int_{\Omega}|\rho(T)|^{2} \mathrm{~d} x+\int_{Q} \frac{1}{\theta^{2}}|\rho|^{2} \mathrm{~d} x \mathrm{~d} t \leq C\left(\int_{Q}|L \rho|^{2} \mathrm{~d} x \mathrm{~d} t+\int_{U}|\rho-P \rho|^{2} \mathrm{~d} x \mathrm{~d} t\right) .
$$

Proof. The proof of Corollary results from three arguments: the $C^{2}$ regularity of $\theta$ on $Q$, the adapted observability inequality (17) and classical properties of the heat equation. Here and in the sequel $C$ denotes a generic positive constant that changes from line to line but that it is independent of the solution.

Since the function $\theta$ is bounded on $Q$, we deduce from (17) that on an interval $(T / 4,3 T / 4)$, we have

$$
\int_{T / 4}^{3 T / 4} \int_{\Omega}|\rho|^{2} \mathrm{~d} x \mathrm{~d} t \leq C\left(\int_{Q}|L \rho|^{2} \mathrm{~d} x \mathrm{~d} t+\int_{U}|\rho-P \rho|^{2} \mathrm{~d} x \mathrm{~d} t\right) \quad \forall \rho \in \mathcal{V} .
$$

Let now $\beta$ be a function satisfying

$$
\beta \in \mathcal{C}^{\infty}([0, T]), \quad 0 \leq \beta \leq 1, \quad \beta=0 \text { on }\left[0, \frac{T}{4}\right], \quad \beta=1 \text { on }\left[\frac{3 T}{4}, T\right] .
$$

Set $\zeta(x, t)=\beta(t) \mathrm{e}^{r t} \rho(x, t)$ where $\rho \in \mathcal{V}$ and $r \in R^{*}$. Then on the one hand we have $\zeta(T)=\mathrm{e}^{r T} \rho(T), \zeta(0)=0$ and on the other hand

$$
\frac{\partial \zeta}{\partial t}-\Delta \zeta+\left(a_{0}-r\right) \zeta=\beta \mathrm{e}^{r t}\left(\frac{\partial \rho}{\partial t}-\Delta \rho+a_{0} \rho\right)+\beta^{\prime} \mathrm{e}^{r t} \rho \quad \text { in } Q .
$$

With this, we multiply the equation (25) by $\zeta$. It follows from the inequality $2 a b \leq a^{2}+b^{2}$ that for every $\mu>0$

$$
\begin{aligned}
\int_{\Omega}|\zeta(T)|^{2} \mathrm{~d} x+\int_{0}^{T} \int_{\Omega}|\nabla \zeta|^{2} \mathrm{~d} x \mathrm{~d} t+\int_{0}^{T} \int_{\Omega}\left(a_{0}-r\right)|\zeta|^{2} \mathrm{~d} x \mathrm{~d} t & \leq \frac{\mu}{2} \int_{0}^{T} \int_{\Omega} \mathrm{e}^{2 r t}|\beta L \rho|^{2} \mathrm{~d} x \mathrm{~d} t \\
& +\frac{1}{2 \mu} \int_{0}^{T} \int_{\Omega}|\zeta|^{2} \mathrm{~d} x \mathrm{~d} t+\int_{0}^{T} \int_{\Omega} \beta^{\prime} \beta \mathrm{e}^{r t}|\rho|^{2} \mathrm{~d} x \mathrm{~d} t .
\end{aligned}
$$


From the properties of $\beta$ and the Poincaré's inequality and for a choice of $r<\left|a_{0}\right|_{L^{\infty}(Q)}$, we have then

$$
\int_{\Omega}|\zeta(T)|^{2} \mathrm{~d} x+\left(1-\frac{C}{2 \mu}\right) \int_{0}^{T} \int_{\Omega}|\nabla \zeta|^{2} \mathrm{~d} x \mathrm{~d} t \leq \frac{\mu}{2} \int_{0}^{T} \int_{\Omega}|L \rho|^{2} \mathrm{~d} x \mathrm{~d} t+C \int_{T / 4}^{3 T / 4} \int_{\Omega}|\rho|^{2} \mathrm{~d} x \mathrm{~d} t .
$$

Finally, as $\zeta(T)=\mathrm{e}^{r T} \rho(T)$, we deduce from (24) that for all $\rho \in \mathcal{V}$ the inequality (23) holds, for a choice of $\mu>C / 2$.

\section{Optimal CONTROL FOR the FOllower}

\subsection{Existence of the optimal control}

Consider now the following symmetric bilinear form

$$
a(\rho, \hat{\rho})=\int_{Q} L \rho L \hat{\rho} \mathrm{d} x \mathrm{~d} t+\int_{U}(\rho-P \rho)(\hat{\rho}-P \hat{\rho}) \mathrm{d} x \mathrm{~d} t .
$$

Thanks to Lemma 1, this bilinear form is a scalar product on $\mathcal{V}$. Let $V$ be the Hilbert space obtained upon taking the closure of $\mathcal{V}$ under the norm:

$$
\rho \mapsto\|\rho\|_{V}=\sqrt{a(\rho, \rho)} .
$$

Remark that the norm $\|\cdot\|_{V}$ is related to the right hand side of the inequality (17). Similarly, the left hand side of (17) leads to the norm

$$
\|\rho\|_{\theta}=\left(\int_{Q} \frac{1}{\theta^{2}}|\rho|^{2} \mathrm{~d} x \mathrm{~d} t\right)^{\frac{1}{2}} .
$$

The completion of $\mathcal{V}$ is the weighted Hilbert space usually denoted by $L_{1 / \theta}^{2}$.

The inequality (17) shows that

$$
\|\rho\|_{\theta} \leq C\|\rho\|_{V}
$$

This inequality extends to $\rho \in V$. This shows that $V$ is continuously imbedded in $L_{1 / \theta}^{2}$.

Let us now consider $h \in L_{\theta}^{2}(Q)$, i.e.:

$$
h \in L^{2}(Q) \quad \text { and } \quad \theta h \in L^{2}(Q) .
$$

From this assumption and thanks to (67) and the Cauchy-Schwarz inequality, we deduce that the linear form defined on $V$ by

$$
\rho \rightarrow \int_{Q} h \rho \mathrm{d} x \mathrm{~d} t+\int_{\Omega} q^{0} \rho(T) \mathrm{d} x
$$

is continuous. By the Lax-Milgram theorem, for any $h \in L_{\theta}^{2}(Q)$ and for any $q^{0} \in L^{2}(\Omega)$, there exits one and only one solution $\rho_{\theta}$ of the variational problem:

$$
\rho_{\theta} \in V, \quad \forall \rho \in V, \quad a\left(\rho_{\theta}, \rho\right)=\int_{Q} h \rho \mathrm{d} x \mathrm{~d} t+\int_{\Omega} q^{0} \rho(T) \mathrm{d} x .
$$


Theorem 1. Assume that (13) and (14) hold. Let $\rho_{\theta}$ be the unique solution of (30) and $P \rho_{\theta}$ be the projection of $\rho_{\theta} \chi_{\omega}$. Set

$$
k_{\theta}=-\left(\rho_{\theta} \chi_{\omega}-P \rho_{\theta}\right)
$$

and

$$
q_{\theta}=L \rho_{\theta} .
$$

Then, the pair $\left(k_{\theta}, q_{\theta}\right)$ is such that (1), (4) and (5) hold. Moreover, we have

$$
\begin{gathered}
\left\|\rho_{\theta}\right\|_{V} \leq C\left[\|\theta h\|_{L^{2}(Q)}+\left\|q^{0}\right\|_{L^{2}(\Omega)}\right], \\
\left\|k_{\theta}\right\|_{L^{2}(U)} \leq C\left[\|\theta h\|_{L^{2}(Q)}+\left\|q^{0}\right\|_{L^{2}(\Omega)}\right], \\
\left\|q_{\theta}\right\|_{W(0, T)} \leq C\left[\|\theta h\|_{L^{2}(Q)}+\left\|q^{0}\right\|_{L^{2}(\Omega)}\right],
\end{gathered}
$$

where $C$ is a positive constant depending only on $\Omega, \omega, a_{0}, T$ and $\mathcal{K}$.

Proof. Since $\rho_{\theta} \in V$ then $k_{\theta}=-\left(\rho_{\theta} \chi_{\omega}-P \rho_{\theta}\right) \in L^{2}(U)$ and $q_{\theta} \in L^{2}(Q)$. Since $P \rho_{\theta} \in \mathcal{K}$ then $k_{\theta}=-\left(\rho_{\theta} \chi_{\omega}-\right.$ $\left.P \rho_{\theta}\right) \in \mathcal{K}^{\perp}$. By direct subtitution in the formulas (26), (30) and (32) it follows

$$
\int_{Q} q_{\theta} L \rho \mathrm{d} x \mathrm{~d} t+\int_{U}\left(\rho_{\theta}-P \rho_{\theta}\right)(\rho-P \rho) \mathrm{d} x \mathrm{~d} t=\int_{Q} h \rho \mathrm{d} x \mathrm{~d} t+\int_{\Omega} q^{0} \rho(T) \mathrm{d} x, \quad \forall \rho \in V .
$$

Taking into account that $P \rho \in \mathcal{K}$, the above identity reduces to

$$
\int_{Q} q_{\theta} L \rho \mathrm{d} x \mathrm{~d} t=\int_{Q} h \rho \mathrm{d} x \mathrm{~d} t-\int_{U}\left(\rho_{\theta}-P \rho_{\theta}\right) \rho \mathrm{d} x \mathrm{~d} t+\int_{\Omega} q^{0} \rho(T) \mathrm{d} x, \quad \forall \rho \in V .
$$

i.e.

$$
\int_{Q} q_{\theta} L \rho \mathrm{d} x \mathrm{~d} t=\int_{Q} h \rho \mathrm{d} x \mathrm{~d} t+\int_{U} k_{\theta} \rho \mathrm{d} x \mathrm{~d} t+\int_{\Omega} q^{0} \rho(T) \mathrm{d} x, \quad \forall \rho \in V .
$$

We show now that $q_{\theta}$ is in fact the weak solution by transposition of a backward heat problem. More precisely, if $\phi \in L^{2}(Q)$, let $p$ be the solution of

$$
\left\{\begin{array}{ccccc}
p^{\prime}-\Delta p+a_{0} p & = & \phi & \text { in } & Q \\
p & = & 0 & \text { on } \Sigma \\
p(0) & = & 0 & \text { in } \Omega .
\end{array}\right.
$$

Then $p \in V$, and thus

$$
\int_{Q} q_{\theta} \phi \mathrm{d} x \mathrm{~d} t=\int_{Q} h p \mathrm{~d} x \mathrm{~d} t+\int_{U} k_{\theta} p \mathrm{~d} x \mathrm{~d} t+\int_{\Omega} q^{0} p(T) \mathrm{d} x .
$$

Therefore $q_{\theta}$ is the weak solution by transposition of problem (1) with $k=k_{\theta}$ (see [15], p. 177). And we know that the solution of this equation is in $W(0, T)$. Therefore $q_{\theta} \in C\left([0, T], L^{2}(\Omega)\right)$. Then multiplying the first 
equation of (1) by $\rho \in \mathcal{V}$ and integrating by parts over $Q$, it follows that for any $\rho \in \mathcal{V}$

$$
\left\{\begin{array}{c}
-\int_{\Omega} q_{\theta}(T) \rho(T) \mathrm{d} x+\int_{\Omega} q_{\theta}(0) \rho(0) \mathrm{d} x+\int_{Q} q_{\theta} L \rho \mathrm{d} x \mathrm{~d} t \\
=\int_{Q} h \rho \mathrm{d} x \mathrm{~d} t+\int_{U} k_{\theta} \rho \mathrm{d} x \mathrm{~d} t .
\end{array}\right.
$$

As $\rho \in \mathcal{V}$, we deduce from (36) that

$$
\int_{\Omega} q_{\theta}(0) \rho(0) \mathrm{d} x=0 \quad \forall \rho \in \mathcal{V} .
$$

Therefore $q_{\theta}(0)=0$ in $\Omega$. Hence, the first statement of Theorem 1 is proved.

It remains to prove the estimates (33)-(35). We set $\rho=\rho_{\theta}$ in (30), it follows from (17) that

$$
\begin{aligned}
a\left(\rho_{\theta}, \rho_{\theta}\right) & =\left\|q_{\theta}\right\|_{L^{2}(Q)}^{2}+\left\|k_{\theta}\right\|_{L^{2}(U)}^{2} \leq\|\theta h\|_{L^{2}(Q)}\left\|\rho_{\theta}\right\|_{\theta}+\left\|q^{0}\right\|_{L^{2}(\Omega)}\left\|\rho_{\theta}(T)\right\|_{L^{2}(\Omega)} \\
& \leq C\left[\|\theta h\|_{L^{2}(Q)}+\left\|q^{0}\right\|_{L^{2}(\Omega)}\right]\left\|\rho_{\theta}\right\|_{V} .
\end{aligned}
$$

Then, from (27) we obtain (33) and thus (34). Finally, (35) is a consequence of (34) and classical properties of the heat equation.

\subsection{Definition of the follower}

The adapted observability inequality (17) shows that the choice of the scalar product on $\mathcal{V}$ is not unique. Thus, there exist infinitely many control functions $k$ such that (1), (4) and (5) hold.

That being, thanks to (30) and Theorem 1, for each scalar product on $\mathcal{V}$ and for each function $h$ in $L_{\theta}^{2}(Q)$, there exists a unique $\rho_{\theta}$ in $V$ and a unique pair $\left(k_{\theta}, q_{\theta}\right)$ such that (1), (4) and (5) hold. Thus, we define the following mappings

$$
\hat{\rho}_{\theta}: h \mapsto \rho_{\theta}, \quad \hat{k}_{\theta}: h \mapsto k_{\theta} \quad \text { and } \quad \hat{q}_{\theta}: h \mapsto q_{\theta} .
$$

Corollary 2. Assume that (13) and (14) hold. The functions $\hat{\rho}_{\theta}, \hat{k}_{\theta}, \hat{q}_{\theta}$ defined in (41) depend linearly on $h$ and are continous from $L_{\theta}^{2}(Q)$ into $V, L^{2}(U)$ and $W(0, T)$ respectively.

Consequently the cost function $J_{\theta}(h)$ is well defined by (6) and thus also the optimization problem (8).

Definition 1. If $h$ is the minimum of the optimization problem (8), we say that $h$ is the optimal leader, $\hat{k}_{\theta}(h)$ is the optimal follower and $\hat{q}_{\theta}(h)$ is the optimal state of the system defined by (1), (4), (5) and (8).

It remains to prove the existence of the optimal leader. This is done in the forthcoming Section 4.

\section{Optimal Control For the Leader}

\subsection{Existence of the leader}

Theorem 2. Under the assumptions (13) and (14), there exists a unique optimal control $\bar{h}_{\theta}$, the so called optimal leader, which is the solution to the problem (8).

Proof. Since $\mathcal{U}_{a d}$ is non empty, from Theorem 1, problem (1), (4) and (5) admits at least one solution. Thus

$$
d=\inf \left\{J_{\theta}(h), \quad h \in \mathcal{U}_{a d}\right\}
$$


is well defined in $\left[0, \infty\left[\right.\right.$. Therefore, if $\left(h_{n}\right)$ is a minimizing sequence, we have

$$
J_{\theta}\left(h_{n}\right) \leq C_{\theta}
$$

where $C_{\theta}$ is a finite constant independent of $n$. Moreover, $h_{n}$ is such that $\left(h_{n}, \hat{k}_{\theta}\left(h_{n}\right), \hat{q}_{\theta}\left(h_{n}\right)\right)$ verifies

$$
\begin{aligned}
& h_{n} \in \mathcal{U}_{a d}, \quad \hat{k}_{\theta}\left(h_{n}\right) \in \mathcal{K}^{\perp}, \quad \hat{q}_{\theta}\left(h_{n}\right) \in W(0, T),
\end{aligned}
$$

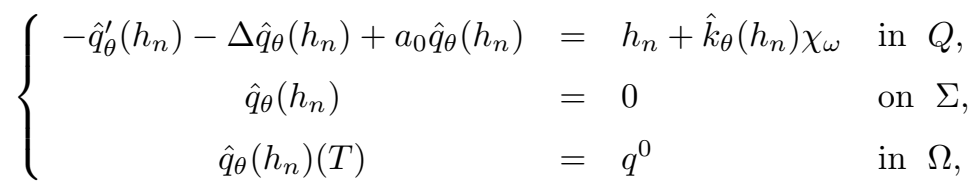

$$
\begin{aligned}
& \hat{q}_{\theta}\left(h_{n}\right)(0)=0 \text { in } \Omega .
\end{aligned}
$$

In a first step, we consider the structure (6) of $J_{\theta}\left(h_{n}\right)$. Since $N>0$, then

$$
\left\|h_{n}\right\|_{\theta} \leq C_{\theta}
$$

Thanks to (34) and (35)

$$
\begin{array}{r}
\left\|\hat{k}_{\theta}\left(h_{n}\right)\right\|_{L^{2}(U)} \leq C\left(\left\|h_{n}\right\|_{\theta}+\left\|q^{0}\right\|_{L^{2}(\Omega)}\right), \\
\mid \hat{q}_{\theta}\left(h_{n}\right) \|_{L^{2}(Q)} \leq C\left(\left\|h_{n}\right\|_{\theta}+\left\|q^{0}\right\|_{L^{2}(\Omega)}\right)
\end{array}
$$

and thus we have

$$
\begin{aligned}
\left\|\hat{k}_{\theta}\left(h_{n}\right)\right\|_{L^{2}(U)} & \leq C_{\theta}, \\
\left\|\hat{q}_{\theta}\left(h_{n}\right)\right\|_{L^{2}(Q)} & \leq C_{\theta} .
\end{aligned}
$$

From the properties of the heat equation we have also

$$
\left\|\hat{q}_{\theta}\left(h_{n}\right)\right\|_{W(0, T)} \leq C_{\theta} .
$$

So we can extract from $\left(h_{n}, \hat{k}_{\theta}\left(h_{n}\right), \hat{q}_{\theta}\left(h_{n}\right)\right)$ a subsequence, still denoted by $\left(h_{n}, \hat{k}_{\theta}\left(h_{n}\right), \hat{q}_{\theta}\left(h_{n}\right)\right)$, such that:

$$
\begin{gathered}
h_{n} \rightarrow \bar{h}_{\theta} \text { weakly in } L_{\theta}^{2}(Q), \quad \bar{h}_{\theta} \in \mathcal{U}_{a d}, \\
\hat{k}_{\theta}\left(h_{n}\right) \rightarrow \bar{k}_{\theta} \text { weakly in } L^{2}(U), \quad \bar{k}_{\theta} \in \mathcal{K}^{\perp},
\end{gathered}
$$

$$
\hat{q}_{\theta}\left(h_{n}\right) \rightarrow \bar{q}_{\theta} \text { weakly in } W(0, T) \text { and strongly in } L^{2}(Q), \quad \bar{q}_{\theta} \in W(0, T) .
$$

Therefore, we can pass to the limit in the system (42)-(44). We obtain

$$
\begin{aligned}
& \bar{h}_{\theta} \in \mathcal{U}_{a d}, \quad \bar{k}_{\theta} \in \mathcal{K}^{\perp}, \quad \bar{q}_{\theta} \in W(0, T)
\end{aligned}
$$

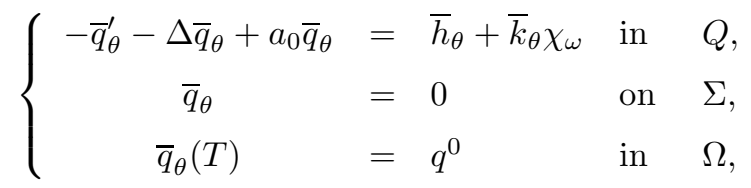

$$
\begin{aligned}
& \bar{q}_{\theta}(0)=0 \text { in } \Omega .
\end{aligned}
$$


Hence, by weak lower semicontinuity of the functional $J_{\theta}$ we deduce that

$$
\liminf J_{\theta}\left(h_{n}\right) \geq \frac{\alpha}{2}\left\|\bar{q}_{\theta}-z_{d}\right\|_{L(Q)^{2}}^{2}+\frac{\beta}{2}\left\|\bar{k}_{\theta}-k_{d}\right\|_{L^{2}(U)}^{2}+\frac{N}{2}\left\|\bar{h}_{\theta}\right\|_{\theta}^{2}
$$

In a second step, we deduce from (30) that $\bar{k}_{\theta}=\hat{k}_{\theta}\left(\bar{h}_{\theta}\right)$ and $\bar{q}_{\theta}=\hat{q}_{\theta}\left(\bar{h}_{\theta}\right)$, i.e.

$$
\left\{\begin{array}{l}
\bar{k}_{\theta}=-\left(\hat{\rho}_{\theta}\left(\bar{h}_{\theta}\right) \chi_{\omega}-P \hat{\rho}_{\theta}\left(\bar{h}_{\theta}\right)\right) \\
\bar{q}_{\theta}=L \hat{\rho}_{\theta}\left(\bar{h}_{\theta}\right)
\end{array}\right.
$$

\subsection{Optimality system for the leader}

Let $\bar{h}_{\theta}$ be the optimal leader, and $\left(\bar{k}_{\theta}, \bar{q}_{\theta}\right)=\left(\hat{k}_{\theta}\left(\bar{h}_{\theta}\right), \hat{q}_{\theta}\left(\bar{h}_{\theta}\right)\right)$ be the corresponding follower-state. The EulerLagrange condition gives

$$
\frac{\mathrm{d}}{\mathrm{d} \lambda} J_{\theta}\left(\bar{h}_{\theta}+\left.\lambda\left(h-\bar{h}_{\theta}\right)\right|_{\lambda=0} \geq 0 \quad \forall h \in \mathcal{U}_{a d}\right.
$$

In order to calculate

$$
\left.\frac{\mathrm{d}}{\mathrm{d} \lambda} J_{\theta}\left(\bar{h}_{\theta}+\lambda w\right)\right|_{\lambda=0}=X(w)
$$

we begin by observing that $\hat{k}_{\theta}$ and $\hat{q}_{\theta}$ depend linearly on $h$ so that

$$
\left\{\begin{array}{l}
\hat{k}_{\theta}\left(\bar{h}_{\theta}+\lambda w\right)=\hat{k}_{\theta}\left(\bar{h}_{\theta}\right)-\lambda\left(\rho(w) \chi_{\omega}-P \rho(w)\right) \\
\hat{q}_{\theta}\left(\bar{h}_{\theta}+\lambda w\right)=\hat{q}_{\theta}\left(\bar{h}_{\theta}\right)+\lambda L \rho(w)
\end{array}\right.
$$

$\rho(w)$ being the unique solution of the following problem

$$
\left\{\begin{array}{l}
\rho(w) \in V \\
a(\rho(w), \rho)=\int_{Q} w \rho \mathrm{d} x \mathrm{~d} t \quad \forall \rho \in V
\end{array}\right.
$$

where $a(.,$.$) and V$ are defined in (26) and (27) respectively. Therefore, we have

$$
X(w)=\int_{Q} \alpha\left(\hat{q}_{\theta}\left(\bar{h}_{\theta}\right)-z_{d}\right) L \rho(w) \mathrm{d} x \mathrm{~d} t-\int_{U} \beta\left(\hat{k}_{\theta}\left(\bar{h}_{\theta}\right)-k_{d}\right)(\rho(w)-P \rho(w)) \mathrm{d} x \mathrm{~d} t+N \int_{Q} \theta^{2} \bar{h}_{\theta} w \mathrm{~d} x \mathrm{~d} t
$$

As $\bar{h}_{\theta}$ is optimal, it follows by Euler-Lagrange's condition in (52) that

$$
X\left(h-\bar{h}_{\theta}\right) \geq 0, \quad \forall h \in \mathcal{U}_{a d}
$$

To simplity these explicit conditions, we introduce the adjoint state defined as follows: for $\rho \in V$, we set

$$
l(\rho)=\int_{Q} \alpha\left(\hat{q}_{\theta}\left(\bar{h}_{\theta}\right)-z_{d}\right) L \rho \mathrm{d} x \mathrm{~d} t-\int_{U} \beta\left(\hat{k}_{\theta}\left(\bar{h}_{\theta}\right)-k_{d}\right)(\rho-P \rho) \mathrm{d} x \mathrm{~d} t .
$$

Then

$$
|l(\rho)| \leq\left\{\left\|\alpha\left(\hat{q}_{\theta}(\bar{h})-z_{d}\right)\right\|_{L^{2}(Q)}^{2}+\left\|\beta\left(\hat{k}_{\theta}\left(\bar{h}_{\theta}\right)-k_{d}\right)\right\|_{L^{2}(U)}^{2}\right\}^{\frac{1}{2}}\left\{\|L \rho\|_{L^{2}(Q)}^{2}+\|\rho-P \rho\|_{L^{2}(U)}^{2}\right\}^{\frac{1}{2}} .
$$

And thus,

$$
|l(\rho)| \leq C\|\rho\|_{V}
$$


In other words, the linear form $\rho \mapsto l(\rho)$ is continuous on $V$. Therefore, there exists a unique solution $\bar{\sigma}_{\theta}$ of the problem

$$
\left\{\begin{array}{l}
\bar{\sigma}_{\theta} \in V \\
a\left(\bar{\sigma}_{\theta}, \rho\right)=l(\rho) \quad \forall \rho \in V .
\end{array}\right.
$$

We shall say that $\bar{\sigma}_{\theta}$ is the associated adjoint state to $\bar{\rho}_{\theta}$ where

$$
\bar{\rho}_{\theta}=\hat{\rho}_{\theta}\left(\bar{h}_{\theta}\right) .
$$

Now, if we choose on the one hand $\rho=\rho(w)$ in (56) where $\rho(w)$ is defined by (53), and on the other one $\rho=\bar{\sigma}_{\theta}$ in (53), then we have

$$
l(\rho(w))=a\left(\bar{\sigma}_{\theta}, \rho(w)\right)=a\left(\rho(w), \bar{\sigma}_{\theta}\right)=\int_{Q} w \bar{\sigma}_{\theta} \mathrm{d} x \mathrm{~d} t
$$

Using the adjoint state $\bar{\sigma}_{\theta}$, we have

$$
X(w)=l(\rho(w))+N \int_{Q} \theta^{2} \bar{h}_{\theta} w \mathrm{~d} x \mathrm{~d} t=\int_{Q} w \bar{\sigma}_{\theta} \mathrm{d} x \mathrm{~d} t+N \int_{Q} \theta^{2} \bar{h}_{\theta} w \mathrm{~d} x \mathrm{~d} t
$$

So, the Euler-Lagrange condition (54) yields

$$
\int_{Q}\left(\bar{\sigma}_{\theta}+N \theta^{2} \bar{h}_{\theta}\right)\left(h-\bar{h}_{\theta}\right) \mathrm{d} x \mathrm{~d} t \geq 0, \quad \forall h \in \mathcal{U}_{a d}
$$

To sum up, we have proved the following result:

Theorem 3. Assume that (13) and (14) hold. Let $\left(\bar{k}_{\theta}, \bar{q}_{\theta}\right) \in L^{2}(U) \times W(0, T)$ be given by (51). The element $\bar{h}_{\theta}$ is the optimal control of the problem (8) if and only if the (unique) solution of

$$
\left\{\begin{array}{l}
\bar{\sigma}_{\theta} \in V, \\
a\left(\bar{\sigma}_{\theta}, \rho\right)=\int_{Q} \alpha\left(\bar{q}_{\theta}-z_{d}\right) L \rho \mathrm{d} x \mathrm{~d} t-\int_{U} \beta\left(\bar{k}_{\theta}-k_{d}\right)(\rho-P \rho) \mathrm{d} x \mathrm{~d} t \quad \forall \rho \in V
\end{array}\right.
$$

satisfies the optimal condition

$$
\int_{Q}\left(\bar{\sigma}_{\theta}+N \theta^{2} \bar{h}_{\theta}\right)\left(h-\bar{h}_{\theta}\right) \mathrm{d} x \mathrm{~d} t \geq 0 \quad \forall h \in \mathcal{U}_{a d}
$$

\section{Discriminating SENTinels}

\subsection{Definition}

Let us remind that the Lions sentinels theory [14] relies on the following three features: the state equation, the observation system and some particular evaluation function: the sentinel itself. More precisely, we consider in the first step the semilinear parabolic equation:

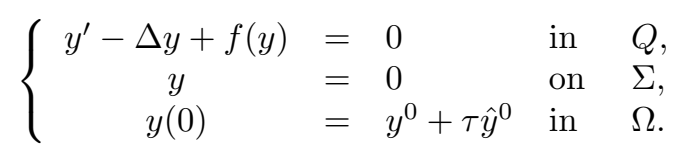

We are interested in systems with data that are not completely known. In the present situation $f: \mathbf{R} \rightarrow \mathbf{R}$ is a given map, and the function $y^{0} \in L^{2}(\Omega)$ is known. However the term, the so called perturbation $\tau \hat{y}^{0}$ is unknown, but is such that

$$
\left\|\hat{y}^{0}\right\|_{L^{2}(\Omega)} \leq 1 \quad \text { and the real number } \tau \text { is small enough. }
$$


In addition to (61), we assume that the non-linearity of $f$ verifies

$$
f(0)=0
$$

and satisfies the following growth condition

$$
\left\{\begin{array}{l}
\left|f\left(s_{1}\right)-f\left(s_{2}\right)-f^{\prime}(0)\left(s_{1}-s_{2}\right)\right| \leq \\
C\left(\left|s_{1}\right|^{p-1}+\left|s_{2}\right|^{p-1}\right)\left|s_{1}-s_{2}\right|, \quad \forall s_{1}, s_{2} \in R
\end{array}\right.
$$

for some $C>0$ and $p>1$ such that

$$
p \leq(d+4) / d
$$

This growth condition is classical (see for instance [19]). Under this growth condition, it is proved in [2], p. 63 that there exists $\alpha>0$ such that when

$$
\left\|y^{0}+\tau \hat{y}^{0}\right\|_{L^{2}(\Omega)} \leq \alpha
$$

the problem $(60)$ admits a unique solution in $C\left([0, T], L^{2}(\Omega)\right)$. Let

$$
y=y\left(\tau, \hat{y}^{0}\right)
$$

be this solution. Therefore, the map

$$
\tau \longmapsto y\left(\tau, \hat{y}^{0}\right) \quad \text { is in } C^{1}\left(R ; C\left([0, T], L^{2}(\Omega)\right) .\right.
$$

Next, consider in the second step the observation process. The observation consists on the knowledge, along some time period, of some function $y_{\text {obs }}$ which is defined on the strip $O \times(0, T)$ over some nonempty open subset $O \subset \Omega$, called observatory. The function $y_{o b s}$ is assumed to be of the form

$$
y_{o b s}=m_{0}+\sum_{i=1}^{M} \beta_{i} m_{i}
$$

where the functions $m_{0}, m_{1}, \ldots, m_{M}$ are given measurements of $y$ in $L^{2}(O \times(0, T))$, but where the real coefficients $\beta_{i}$ are unknown. We assume that $\beta_{i}$ are small. We refer to the terms $\beta_{i} m_{i}$ as the interference terms. We can assume without loss of generality that

$$
\text { the functions } m_{i} \text { are linearly independent. }
$$

Finally, we introduce now the notion of sentinel. Let $h_{0}$ be a given function on $O \times(0, T)$ such that

$$
h_{0} \geq 0, \quad \int_{0}^{T} \int_{O} h_{0} \mathrm{~d} x \mathrm{~d} t=1
$$

Moreover let $\omega$ be an open and non empty subset of $\Omega$. For any control function $w \in L^{2}(\omega \times(0, T))$, set

$$
S(\tau)=\int_{0}^{T} \int_{O} h_{0} y\left(\tau, \hat{y}^{0}\right) \mathrm{d} x \mathrm{~d} t+\int_{0}^{T} \int_{\omega} w y\left(\tau, \hat{y}^{0}\right) \mathrm{d} x \mathrm{~d} t .
$$


The role of the function $w$ appears in the following definition. We shall say that $S$ defines a discriminating sentinel (for the system (60), (67) and (69)) if there exists $w$ such that the functional $S$ satisfies the following conditions:

(i) $S$ is stationary at first order with respect to the missing terms $\tau \hat{y}^{0}$, that is

$$
\frac{\partial S}{\partial \tau}(0)=0 \quad \forall \hat{y}^{0}
$$

(ii) $S$ is stationary with respect to the interference terms $\beta_{i} m_{i}$, that is

$$
\int_{0}^{T} \int_{O} h_{0} m_{i} \mathrm{~d} x \mathrm{~d} t+\int_{0}^{T} \int_{\omega} w m_{i} \mathrm{~d} x \mathrm{~d} t=0, \quad 1 \leq i \leq M .
$$

Remark 4. At this point, some comments must be made.

(1) The original Lions's discriminating sentinel $S$ corresponds to the case where $\omega=O$ and the norm $\|w\|_{L^{2}(\omega \times(0, T))}$ is minimal among control functions $w$ in $L^{2}(\omega \times(0, T))$ which satisfy the above conditions (71)-(72). Therefore, the previous definition introduces a generalization of Lions's discriminating sentinel to the case where the observation and the control have their supports in two different open subsets. This point of view with $w$ of minimal norm is considered in [16].

(2) The support $\operatorname{supp}\left(m_{i}\right)$ of functions $m_{i}$ is assumed to be included in $O$. Suppose $\omega \cap O=\emptyset$. Then, automatically $\int_{0}^{T} \int_{\omega} w m_{i} \mathrm{~d} x \mathrm{~d} t=0$. Therefore, it suffices to choose $h_{0}$ such that $h_{0}$ is orthogonal to each $m_{i}$ and then (72) would be readily verified. Therefore, for all $\omega$ we can neglect the part of $\omega$ which is out of $O$. So, without loss of generality, it may be assumed that

$$
\omega \subset O .
$$

\subsection{Equivalence to the null-controllability}

Here it will be shown that the existence of such a control function $w$ satisfying (71)-(72) is equivalent to the null-controllability property for a system with constrained control. First, we denote by $\bar{y}$ the solution of problem (60) for $\tau=0$ and we assume that $\bar{y}$ can be computed in practice. Next, we consider the function $y_{\tau}$ defined by

$$
y_{\tau}=\left.\frac{\mathrm{d}}{\mathrm{d} \tau} y\left(\tau, \hat{y}^{0}\right)\right|_{\tau=0} .
$$

The function $y_{\tau}$ is the solution of the linearized problem

$$
\left\{\begin{array}{cllll}
y_{\tau}^{\prime}-\Delta y_{\tau}+f^{\prime}(\bar{y}) y_{\tau} & = & 0 & \text { in } & Q, \\
y_{\tau} & = & 0 & \text { on } & \Sigma, \\
y_{\tau}(0) & = & y^{0} & \text { in } & \Omega
\end{array}\right.
$$

where $f^{\prime}(\bar{y})$ denotes the derivative of $f$ on $\bar{y}$. Thanks to (63), problem (75) admits a unique solution $y_{\tau}$.

We now consider the stationary condition (71). It holds if and only if

$$
\int_{0}^{T} \int_{O} h_{0} y_{\tau} \mathrm{d} x \mathrm{~d} t+\int_{0}^{T} \int_{\omega} w y_{\tau} \mathrm{d} x \mathrm{~d} t=0 \quad \forall \hat{y}^{0} \quad: \quad\left\|\hat{y}^{0}\right\|_{L^{2}(\Omega)} \leq 1
$$


In order to transform the equation (76), introduce now the classical adjoint state. More precisely, consider the solution $q=q(x, t)$ of the linear problem

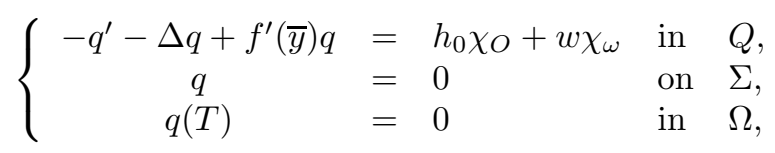

where $\chi_{O}$ and $\chi_{\omega}$ are the characteristic functions for the open sets $O$ and $\omega$ respectively. As for the problem (75), problem (77) admits a unique solution $q$. The so called adjoint state $q$ depends on the unknown $w$ and its usefulness comes from the following observation.

First, multiply both members of the differential equation in (77) by $y_{\tau}$, and integrate by parts over $Q$ :

$$
\int_{0}^{T} \int_{O} h_{0} y_{\tau} \mathrm{d} x \mathrm{~d} t+\int_{0}^{T} \int_{\omega} w y_{\tau} \mathrm{d} x \mathrm{~d} t=\int_{\Omega} q(0) \hat{y}^{0} \mathrm{~d} x \quad \forall \hat{y}^{0}, \quad\left\|\hat{y}^{0}\right\|_{L^{2}(\Omega)} \leq 1 .
$$

Thus, the condition (71) (or (76)) holds if and only if

$$
q(0)=0
$$

Then, consider the constraints $(72)$. Let $\mathcal{K}$ be the vector subspace generated in $L^{2}(\omega \times(0, T))$ by the $M$ independent functions $m_{i} \chi_{\omega}$. There is a unique $k_{0} \in \mathcal{K}$ such that

$$
\int_{0}^{T} \int_{O} h_{0} m_{i} \mathrm{~d} x \mathrm{~d} t+\int_{0}^{T} \int_{\omega} k_{0} m_{i} \mathrm{~d} x \mathrm{~d} t=0,1 \leq i \leq M .
$$

In other words, the condition (72) holds if and only if

$$
w-k_{0}=k \in \mathcal{K}^{\perp} .
$$

The above considerations show that finding the control $w$ such that the functional $S$ satisfies $(71)-(72)$ is equivalent to finding the control $k$ such that the pair $(k, q)$ satisfies the following system

$$
\begin{aligned}
& k \in \mathcal{K}^{\perp}, \quad q \in W(0, T),
\end{aligned}
$$

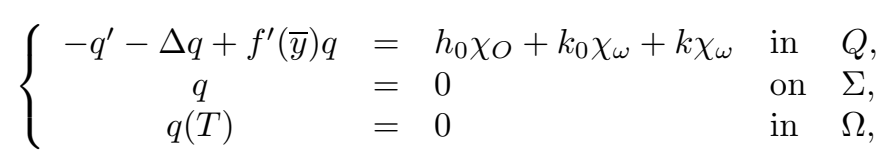

$$
\begin{aligned}
& q(0)=0 \text { in } \Omega .
\end{aligned}
$$

We see that (81)-(83) is exactly the problem (1), (4) and (5) when $a_{0}=f^{\prime}(\bar{y}), h=h_{0} \chi_{O}+k_{0} \chi_{\omega}$ and $q^{0}=0$. So, we can apply the results obtained in the previous sections. Indeed, observe first that $\mathcal{K}$ is finite dimensional. Now, assume that $\mathcal{K}$ and $h=h_{0} \chi_{O}+k_{0} \chi_{\omega}$ are such that (14) and (29) hold respectively. We assume also (73). Now, let $\left(\rho_{\theta}, k_{\theta}, q_{\theta}\right)$ be defined as in Theorem 1. Then

$$
k_{\theta}=-\left(\rho_{\theta} \chi_{\omega}-P \rho_{\theta}\right),
$$

and the sentinel is defined by

$$
S(\tau)=\int_{0}^{T} \int_{O} h_{0} y\left(\tau, \hat{y}^{0}\right) \mathrm{d} x \mathrm{~d} t+\int_{0}^{T} \int_{\omega}\left(k_{0}-\left(\rho_{\theta}-P \rho_{\theta}\right)\right) y\left(\tau, \hat{y}^{0}\right) \mathrm{d} x \mathrm{~d} t .
$$


Now, if $h_{0}$ is subject to some constraints, for example if $h_{0}$ remains in a suitable non empty convex set, then it is natural to introduce the control point of view. For instance, we would like to choose $h_{0}$ such that the adjoint state $q$ of the system does not move too far from a given state. So, we can use the previous notion of leader and follower. Thus, given $z_{d} \in L^{2}(Q), k_{d} \in L^{2}(U)$, let $\bar{h}_{\theta}$ be the optimal solution of (8), $\bar{\rho}_{\theta}$ the corresponding solution of (30), and define $\bar{S}_{\theta}$ by

$$
\bar{S}_{\theta}(\tau)=\int_{0}^{T} \int_{O} h_{0} y\left(\tau, \hat{y}^{0}\right) \mathrm{d} x \mathrm{~d} t+\int_{0}^{T} \int_{\omega}\left(k_{0}-\left(\bar{\rho}_{\theta}-P \bar{\rho}_{\theta}\right) y\left(\tau, \hat{y}^{0}\right) \mathrm{d} x \mathrm{~d} t .\right.
$$

This is the optimal sentinel for the system (60), (67), (69), (6)-(8).

Acknowledgements. The author wishes to express his gratitude to the referee for his many helpful suggestions.

\section{REFERENCES}

[1] V. Barbu, Exact controllability of the superlinear heat equation. Appl. Math. Optim. 42 (2000) 73-89.

[2] T. Cazenave and A. Haraux, Introduction aux Problèmes d'Evolution Semi-Linéaires, Collection Mathématiques et Applications de la SMAI. Éditions Ellipses, Paris (1991).

[3] R. Dorville, Sur le contrôle de quelques problèmes singuliers associés à l'équation de la chaleur. Ph.D. thesis, Université des Antilles et de la Guyane (2004).

[4] R. Dorville, O. Nakoulima and A. Omrane, Low-regret control for singular distributed systems: The backwards heat ill-posed problem. Appl. Math. Lett. 17 (2004) 549-552.

[5] A. Doubova, A. Osses and J.P. Puel, Exact controllability to trajectories for semilinear heat equations with discontinuous diffusion coefficients. ESAIM: COCV 8 (2002) 621-661.

[6] C. Fabre, J.P. Puel and E. Zuazua, Approximate controllability of the semilinear heat equation. Proc. Royal Soc. Edinburg 125A (1995) 31-61.

[7] E. Fernández-Cara, Nul controllability of the semilinear heat equation. ESAIM: COCV 2 (1997) 87-103.

[8] E. Fernández-Cara and S. Guerrero, Global Carleman inequalities for parabolic systems and applications to controllability. SIAM J. Control Optim. 45 (2006) 1395-1446.

[9] E. Fernández-Cara and E. Zuazua, The cost of approximate controllability for heat equations: the linear case. Adv. Differ. Equ. 5 (2000) 465-514.

[10] A. Fursikov and O.Yu. Imanuvilov, Controllability of evolution equations, Lecture Notes. Research Institute of Mathematics, Seoul National University, Korea (1996).

[11] O.Yu. Imanuvilov, Controllability of parabolic equations. Sbornik Math. 186 (1995) 879-900.

[12] G. Lebeau and L. Robbiano, Contrôle exacte de l'équation de la chaleur. Comm. Part. Diff. Eq. 20 (1995) 335-356.

[13] J.L. Lions, Contrôle optimal de systèmes gouvernés par des équations aux dérivées partielles. Dunod, Gauthier-Villars, Paris (1968).

[14] J.L. Lions, Sentinelles pour les systèmes distribués à données incomplètes. Masson, Paris (1992).

[15] J.L. Lions and M. Magenes, Problèmes aux limites non homogènes et applications. Vols. 1 et 2, Dunod, Paris (1988).

[16] O. Nakoulima, Contrôlabilité à zéro avec contraintes sur le contrôle. C. R. Acad. Sci. Paris Ser. I Math. 339 (2004) 405-410.

[17] D.L. Russell, A unified boundary controllability theory for hyperbolic and parabolic partial differential equations. Stud. App. Math. 52 (1973) 189-212.

[18] E. Zuazua, Exact boundary controllability for the semilinear wave equation. Non linear Partial Diff. Equ. Appl. 10 (1989) 357-391.

[19] E. Zuazua, Finite dimensional null controllability for the semilinear heat equation. J. Math. Pures Appl. 76 (1997) $237-264$.

[20] E. Zuazua, controllability of partial differential equations and its semi-discrete approximations. Discrete Continuous Dynam. Syst. 8 (2002) 469-513. 